\title{
Gray Level Co-Occurrence Matrix (GLCM) and Gabor Features Based No-Reference Image Quality Assessment for Wood Images
}

\author{
Heshalini Rajagopal ${ }^{1,2}$, Norrima Mokhtar ${ }^{1}$, Anis Salwa Mohd Khairuddin', Wan Khairunizam³, \\ Zuwairie Ibrahim ${ }^{4}$, Asrul Bin Adam ${ }^{4}$ and Wan Amirul Bin Wan Mohd Mahiyidin ${ }^{1}$ \\ ${ }^{1}$ Department of Electrical Engineering, Faculty of Engineering, University of Malaya, Malaysia \\ ${ }^{2}$ Department of Electrical and Electronic Engineering, Manipal International University, Malaysia \\ ${ }^{3}$ School of Mechatronic Engineering, University of Malaysia Perlis, Malaysia \\ ${ }^{4}$ College of Engineering, University of Malaysia Pahang, Malaysia \\ 'heshalini@gmail.com
}

\begin{abstract}
Image Quality Assessment (IQA) is an imperative element in improving the effectiveness of an automatic wood recognition system. There is a need to develop a No-Reference-IQA (NR-IQA) system as a distortion free wood images are impossible to be acquired in the dusty environment in timber factories. Therefore, a Gray Level CoOccurrence Matrix (GLCM) and Gabor features-based NR-IQA, GGNR-IQA algorithm is proposed to evaluate the quality of wood images. The proposed GGNR-IQA algorithm is compared with a well-known NR-IQA, Blind/Referenceless Image Spatial Quality Evaluator (BRISQUE) and Full-Reference-IQA (FR-IQA) algorithms, Structural Similarity Index (SSIM), Multiscale SSIM (MS-SSIM), Feature SIMilarity (FSIM), Information Weighted SSIM (IW-SSIM) and Gradient Magnitude Similarity Deviation (GMSD). Results shows that the GGNR-IQA algorithm outperforms the NR-IQA and FR-IQAs. The GGNR-IQA algorithm is beneficial in wood industry as a distortion free reference image is not required to pre-process wood images.
\end{abstract}

Keywords: Wood images, GLCM, Gabor, GGNR-IQA, NR-IQA

\section{INTRODUCTION}

Wood is extensively used for furniture, building construction and paper production ${ }^{[1]}$. There are various types of wood and each of them has different attributes with regard to its formation, thickness, colour and texture ${ }^{[2]}$. These varying characteristics defines their ideal usages and economic values $^{[3]}$. As the price and characteristics of every wood species differs, misclassification may cause financial losses. Hence, there is a need to identify different wood species accurately.

Conventionally, the recognition of wood species is performed manually by human subjects ${ }^{[4]}$. However, this practice is time and cost consuming to the lumber industry. Hence, several automatic wood species recognition systems have been developed ${ }^{[1,2,5,6]}$. The efficiency of automatic wood recognition systems can be improved by using superior quality microscopy images which are commonly enhanced to improve the rate of successful wood species recognition. Nevertheless, the image enhancement processes consume extra computational time, and could cause a checkerboard artefact to the wood images ${ }^{[7]}$. In addition, the dusty and dark environment in timber factories could degrade the quality of the image acquired ${ }^{[8]}$. Thus, an appropriate image quality assessment (IQA) algorithm is required to assess the acquired wood images prior to feeding it to any automatic wood recognition system.

IQA can be categorised into subjective and objective evaluations. Subjective evaluation is the scores given by human subjects based on their judgment on the image quality. While, objective evaluation is done based on numerical methods to determine the quality of the images. Even though, subjective evaluation is the benchmark of IQA it is impracticable in an industrial environment as it is time and cost consuming. Therefore, it is necessary to develop an objective evaluation procedures that is capable to imitate subjective IQA evaluation ${ }^{[9]}$.

Objective evaluation can be categorised into FullReference-IQA (FR-IQA), Reduced Reference-IQA (RRIQA) and No-Reference/Blind IQA (NR-IQA) ${ }^{[10,11]}$. FRIQA uses the reference image fully to assess the images 
whereas RR-IQA uses the reference images partially. In contrast, NR-IQA assesses an image without using a reference image. NR-IQA is the most appropriate algorithm to evaluate the quality of the wood images as it may be impossible to obtain high quality images in the dusty and dark setting of lumber factories. Therefore, we propose the Gray Level Co-Occurrence Matrix (GLCM) and Gabor features-based NR-IQA, GGNR-IQA algorithm to evaluate wood images.

The GLCM and Gabor features are widely used in wood species recognition system ${ }^{[5,12-14]}$. The proposed GGNRIQA algorithm is compared with a commonly utilized NRIQA, Blind/Referenceless Image Spatial Quality Evaluator (BRISQUE), and FR-IQAs namely, Structural Similarity Index (SSIM) ${ }^{[15]}$, Multiscale SSIM (MS-SSIM) ${ }^{[15]}$, Feature Similarity (FSIM) ${ }^{[16]}$, Information Weighted SSIM (IWSSIM $)^{[17]}$ and Gradient Magnitude Similarity Deviation $(\mathrm{GMSD})^{[18]}$. The performances of the GGNR-IQA, BRISQUE and FR-IQAs are evaluated by using the Pearson Linear Correlation Coefficient (PLCC) and Root Mean Squared Error (RMSE) computed between the human mean opinion scores (MOS) and the algorithms.

\section{METHODS}

\subsection{Training and Testing Database}

An SVR model is trained with the GLCM and Gabor features calculated for normalized wood images with the human MOS which are obtained from the subjective evaluation for wood images. The MOS, GLCM and Gabor features are utilized as the training and testing database to obtain an optimized SVR model

\subsubsection{Wood Images}

Ten wood images from various wood genus, as shown in Fig. 1 were chosen. The images were acquired from a wood database: https://www.wood-database.com/ [19]. The ten reference images were distorted by Gaussian white noise and motion blur. These two types of distortions usually occur in the industrial setting. Generally, the wood images are exposed to Gaussian white noise due to the poor illumination and heat in the lumber mill while acquiring the wood images ${ }^{[8,20]}$. On the other hand, wood images are exposed to motion blur when there is a relative motion between the wood slice and camera ${ }^{[6]}$.

These distortions degrade the quality of the wood images where the features of the pores on the wood texture may not be discerned. Hence, this may lead to misclassification of the wood genus as the feature extractor may not obtain distinctive features from the wood images
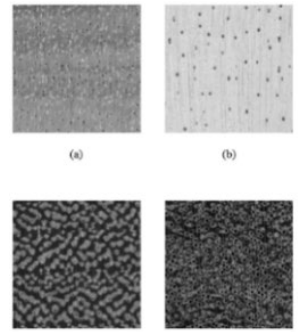

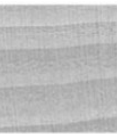

(s)

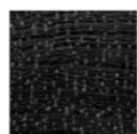

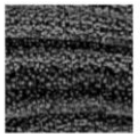
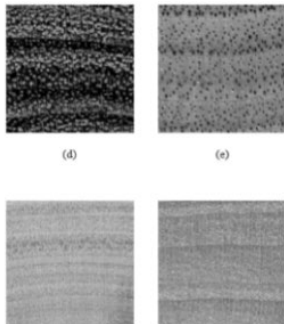

(a)

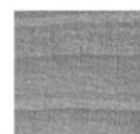

() efficiently ${ }^{[21]}$.Nine modulations of Gaussian white noise with standard deviation, $\sigma_{G N}$ and motion blur with standard deviation, $\sigma_{M B}$ were added to the reference images, i.e.: $\sigma_{G N}$ $=10,20,30,40,50,60,70,80$ and 90 for Gaussian white noise and $\sigma_{M B}=2,4,6,8,10,12,14,16$ and 18 for motion blur.

Fig 1. Ten wood images used as reference images (a) Turraeanthus africanus, (b) Ochroma pyramidale, (c) Tilia americana, (d) Cordia spp., (e) Juglans cinerea, (f) Vouacapoua americana, (g) Dipterocarpus spp., (h) Swartzia Cubensis, (i) Cordia spp., (j) Cornus florida

\subsubsection{GLCM and Gabor Features}

First, Mean Subtracted Contrast Normalized (MSCN), $\hat{I}(m, n)$ is calculated from the wood image, $I(m, n)$ using Eq. (1) ${ }^{[22]:}$

$$
\hat{I}(m, n)=\frac{I(m, n)-\mu(m, n)}{\sigma(m, n)+1}
$$

where $\mu(m, n)$ and $\sigma(m, n)$ denote the mean and variance of wood image, $I(m, n)$, respectively $, m \in 1,2, \ldots, M, n \in$ $1,2, \ldots, N$ are spatial indices while $M$ represents the height and $N$ represents width of image, $I(m, n)$.

The mean, $\mu(m, n)$ and variance, $\sigma(m, n)$ of the wood image are computed using Eq. (2) and Eq. (3), respectively [22]:

$\mu(m, n)=\sum_{k=-K}^{K} \sum_{l=-L}^{L} w_{k, l} I_{k, l}(m, n)$

$\sigma(m, n)=\sqrt{\sum_{k=-K}^{K} \sum_{l=-L}^{L} w_{k, l}\left(I_{k, l}(m, n)-\mu(m, n)\right)^{2}}$

where $w=\left\{w_{k, l} \mid k=-K, \ldots, K, l=-L, \ldots, L\right\}$ is a 2 dimension (2D) circularly-symmetric Gaussian weighting function that is sampled out to three standard deviations and rescaled to unit volume, and $K$ and $L$ represent the window sizes.

The MSCN coefficients, $\hat{I}(m, n)$ highlights the main features of the wood images such as pores and grains, with few low-energy residual object boundaries ${ }^{[23]}$. Therefore, the MSCN is used to compute the GLCM and Gabor features instead of the image, $I(m, n)$. Next, two types of features 
namely, GLCM and Gabor features were incorporated in this study.

\subsubsection{GLCM Features}

The GLCM depicts second order statistical analysis of an image by analyzing how often the pairs of pixels which consist of specific values and spatial relationship take place in an image. The probability, $p(m, n)$ is computed using Eq. (4) ${ }^{\text {[24]: }}$

$p(m, n)=\{C(m, n) \mid(d, \theta)\}$

where $d$ is the inter-pixels displacement distance, $\theta$ denotes orientation and $C(m, n)$ denotes the frequency of gray level occurrence in MSCN of the image, $\hat{I}(m, n)$. Four statistical textures such as contrast, correlation, energy, and homogeneity were extracted from the GLCM matrix.

Contrast calculates the local variations in the gray-level co-occurrence matrix and is defined as Eq. (5) ${ }^{[24]}$ :

Contrast $=\sum_{m, n}|m-n|^{2} p(m, n)$

Correlation computes the joint probability occurrence of the specified pixel pairs and is defined as Eq. (6) ${ }^{[24]}$ :

Correlation $=\sum_{m, n} \frac{(m-\mu m)(n-\mu n) p(m, n)}{\sigma_{m} \sigma_{n}}$

Energy calculates the sum of squared components in the GLCM. It is also known as uniformity or the angular second moment. The energy parameter is computed as Eq. (7) ${ }^{[24]}$ : Energy $=\sum_{m, n} p(m, n)^{2}$

Homogeneity calculates the closeness of the distribution of elements in the GLCM to the GLCM diagonal and is computed as Eq. (8) ${ }^{[24]}$ :

Homogeneity $=\sum_{m, n} \frac{p(m, n)}{1+|m-n|}$

These 4 parameters were computed at four directions, $0^{\circ}$, $45^{\circ}, 90^{\circ}$ and $135^{\circ}$ and this form 16 GLCM features.

\subsubsection{Gabor Features}

The 2D Gabor function which represents the spatial summation properties of simple cells in the visual cortex and it is defined as Eq. (9) ${ }^{[25]}$ :

$$
g(x, y ; \lambda, \theta, \psi, \sigma, \gamma)=\exp \left(-\frac{x^{2}+\gamma^{2} y^{2}}{2 \sigma^{2}}\right) \cos \left(2 \pi \frac{x \prime}{\lambda}+\psi\right)
$$

Where

$x^{\prime}=x \cos \theta+y \sin \theta$

$y^{\prime}=-x \sin \theta+y \cos \theta$

$\lambda$ denotes the wavelength of the sinusoidal factor, $\theta$ denotes the orientation of the normal to the parallel stripes of a Gabor function, $\psi$ represents the phase offset, $\sigma$ represents the standard deviation of the Gaussian envelope and $\gamma$ represents the spatial aspect ratio.
The computational models of 2D Gabor filters are defined in Eq. (12) and (13) ${ }^{[25]}$ :

$h_{e}=g(x, y) \cos (2 \pi f(x \cos \theta+y \sin \theta))$

$h_{o}=g(x, y) \sin (2 \pi f(x \cos \theta+y \sin \theta))$

Where $h_{e}$ and $h_{o}$ represents the even symmetric and odd symmetric Gabor filters, respectively and $g(x, y)$ represents the isotropic Gaussian function and is computed as Eq. (14) [25]:

$g(x, y)=\frac{1}{\sqrt{2 \pi \sigma^{2}}} \exp \left(-\frac{x^{2}+y^{2}}{2 \sigma^{2}}\right)$

And the spatial frequency response of the Gabor functions, $f$ is as shown in Eq. (15) ${ }^{[25]}$ :

$f=N / P$

Where $N$ denotes the size of the kernel and $P$ denotes period in pixel.

In this study, wavelength, $\lambda$ is in increasing powers of two starting from $4 / \sqrt{2}$ up to the hypotenuse length of the input image ${ }^{[26]}$ and this produces 7 Gabor features. The 7 Gabor features were then computed in four orientations, $0^{\circ}$, $45^{\circ}, 90^{\circ}$ and $135^{\circ}$, similar to the GLCM computations. This forms 28 features Gabor features. In total, the 16 GLCM and 28 Gabor features were combined and this forms 44 features. These 44 features were calculated using the MSCN coefficients, $\hat{I}(m, n)$ and are used to train SVR.

\subsubsection{MOS}

The MOS values were obtained from subjective evaluation participated by 10 students aged between 20-25 years from Manipal International University (MIU), Malaysia. The evaluation was carried out as per the procedures suggested in Rec. ITU-R BT.500-11 ${ }^{[27]}$ where it was performed in an office environment using a 21-inch LED computer screen.

Simultaneous Double Stimulus for Continuous Evaluation (SDSCE) approach was used in this evaluation ${ }^{[27,28]}$ where the reference and distorted images are shown side-by-side on the computer screen and each subject compares the quality of the images displayed on the right side with its reference image (left side) to evaluate the displayed image.

The score given by the human subjects are either Excellent (5), Good (4), Fair (3), Poor (2) or Bad (1) for each image displayed. The evaluation process takes 15 to 20 minutes for each subject. The scores obtained from the subjects were averaged to convert them to the $\operatorname{MOS}^{[29]}$. These MOS values are also used to train SVR.

\subsubsection{Support Vector Machine Regression (SVR)}

$\in-$ SVR $^{[30]}$ is trained using MOS and 44 GLCM and Gabor features of wood images in this study. The 44 image

(C) The 2021 International Conference on Artificial Life and Robotics (ICAROB2021), January 21 to 24, 2021 
features (GLCM and Gabor features) calculated for the wood images are mapped to the MOS values of the corresponding wood images.

The 44 GLCM and Gabor features and MOS of wood images were randomly split into training and testing sets where $80 \%$ of the 44 features and MOS values were used to

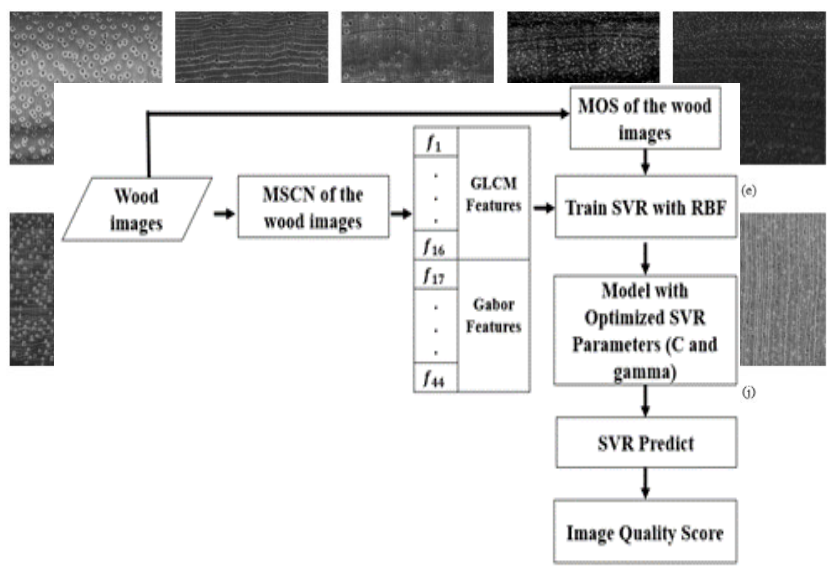

train the SVR model to obtain an SVR model with optimized parameters and $20 \%$ were utilized to evaluate the optimized SVR model.

The flow diagram of the proposed GGNR-IQA is shown in Fig. 2. The performance of GGNR-IQA was evaluated using PLCC ${ }^{[31]}$ and $\mathrm{RMSE}^{[32]}$ calculated between 1000 iterations were performed on the training and testing of the SVR model to obtain an optimized SVR model. The cost parameter, C, and width parameter, $g$, of the optimized SVR model are 32768 and 0.125 , respectively.

Fig 2. Flow Diagram of the Proposed GGNR-IQA

\subsection{Performance Evaluation}

The proposed GGNR-IQA is compared with a wellknown NR-IQA algorithm, BRISQUE and five FR-IQAs ${ }^{[21]}$ : Structural Similarity Index (SSIM ${ }^{[15]}$, Multiscale SSIM $(\mathrm{MS}-\mathrm{SSIM})^{[15]}$, Feature Similarity (FSIM) ${ }^{[16]}$, Information Weighted SSIM (IW-SSIM) $)^{[17]}$ and Gradient Magnitude Similarity Deviation (GMSD) ${ }^{[18]}$.

The performance of the GGNR-IQA, BRISQUE and FR-IQAs is assessed using PLCC and RMSE ${ }^{[28]}$ values calculated between these algorithms and MOS.

\section{RESULTS AND DISCUSSIONS}

The efficiency of the GGNR-IQA was further assessed using a second dataset which was generated from the same wood image database ${ }^{[19]}$. This second dataset was produced using ten reference images acquired from ten various wood genus as shown in Fig. 3.

Fig 3. Reference wood images in the second dataset (a) Julbernardia pellegriniana,, (b) Dalbergia cultrate, (c) Dalbergia retusa, (d) Dalbergia cearensis, (e) Guaiacum officinale, (f) Swartzia spp., (g) Dalbergia spruceana, (h)

Dalbergia sissoo, (i) Swartzia benthamiana and (j) Euxylophora paraensis

These reference images were added with the similar distortion type (Gaussian white noise and motion blur) and modulations as the training and testing database. This means that the second dataset includes 10 reference images and 180 distorted images.

\subsection{Relationship Between MOS and Quality of Image with Different Distortion Modulations}

Fig. 4 (a) and (b) shows the correlation between MOS and nine distortion modulations of Gaussian white noise and motion blur. Lower MOS values show lower image quality which is caused by higher distortion modulation. On the other hand, higher MOS values represent higher image quality which is generated by lower distortion modulation. Based on Fig. 4 (a) and (b), the MOS decreases as the distortion modulation increases. This means that all the human subjects could discern the images distorted with the various modulations of Gaussian white noise and motion blur.

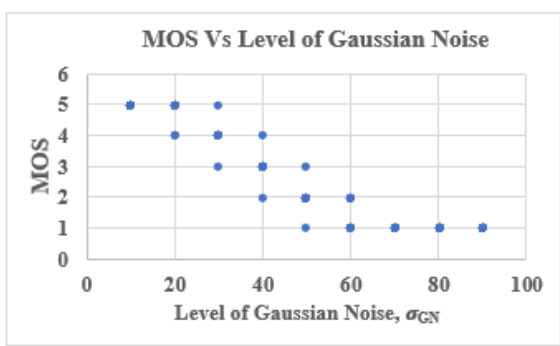

(a)

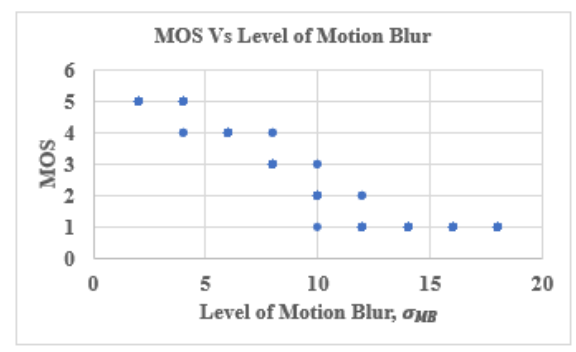

(b) 
Fig 4. Scatter Plot of MOS versus nine distortion modulations of (a) Gaussian White Noise and (b) Motion Blur

\subsection{Correlation between GGNR-IQA, BRISQUE and FR-IQAs Algorithms and MOS}

The PLCC and RMSE values calculated between GGNR-IQA, BRISQUE, five FR-IQA algorithms: Structural Similarity Index (SSIM), Multiscale SSIM (MSSSIM), Feature SIMilarity (FSIM), Information Weighted SSIM (IW-SSIM), Gradient Magnitude Similarity Deviation (GMSD) and MOS for Gaussian white noise, motion blur and overall images are shown in Fig. 5 and Fig. 6, respectively. The most suitable IQA for wood images is expected to have the highest PLCC and lowest RMSE values.

Based on Fig. 5 and Fig. 6, the GGNR-IQA has the highest PLCC and lowest RMSE values for Gaussian white noise, motion blur and the overall database compared to the BRISQUE and FR-IQAs. This shows that the GGNR-IQA algorithm outperforms BRISQUE and all the five FR-IQAs.

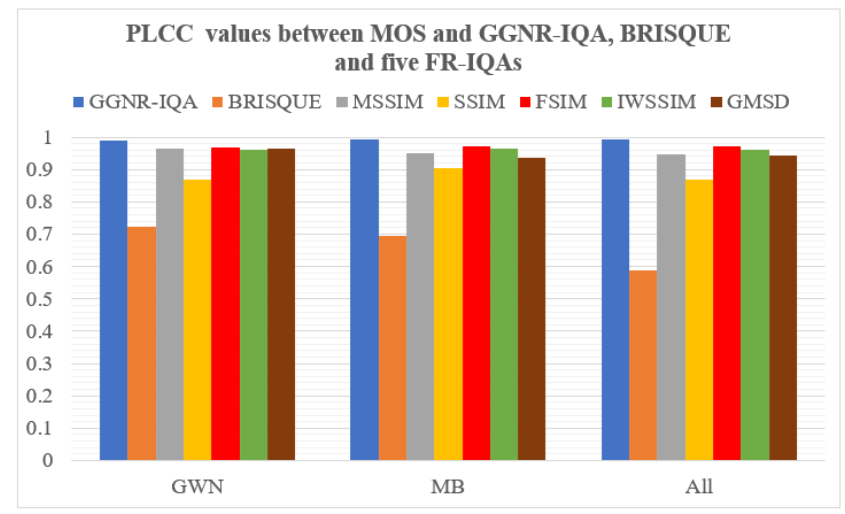

Fig. 5. PLCC values between GGNR-IQA, BRISQUE, FRIQAs and MOS

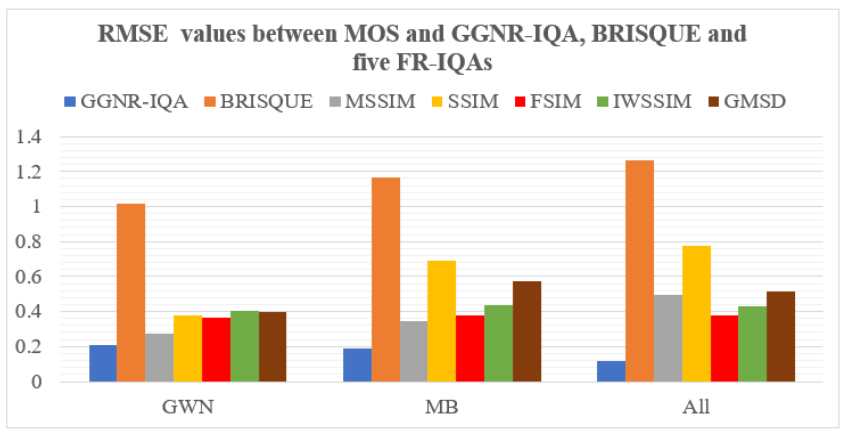

Fig. 6. RMSE values between GGNR-IQA, BRISQUE, FR-IQAs and MOS

\section{CONCLUSIONS}

A NR-IQA algorithm, GGNR-IQA was proposed to assess wood images prior to feeding the image to wood species classification and recognition system. The proposed GGNR-IQA algorithm was trained using GLCM, Gabor features and MOS obtained from wood images. The performance of the GGNR-IQA algorithm was assessed by comparing the PLCC and RMSE values calculated between GGNR-IQA, BRISQUE, five FR-IQA algorithms and MOS. PLCC and RMSE values showed that the GGNR-IQA algorithm outperforms BRISQUE and all the five FR-IQAs.

This shows that the GGNR-IQA algorithm could assess the quality of wood images accurately. In addition, the proposed GGNR-IQA algorithm would not require a distortion free reference image to determine the quality of the wood images. This is beneficial especially when it is impossible to obtain a distortion free reference image in the dusty environment of lumber mill.

\section{REFERENCES}

[1] Shivashankar S. Automatic Wood Classification using a Novel Color Texture Features. Int $J$ Comput Appl. 2018;180(27):34-38.

[2] Barmpoutis P, Dimitropoulos K, Barboutis I, Grammalidis N. Wood species recognition through multidimensional texture analysis. Comput Electron Agric. 2018;144:241-248. doi:10.1016/j.compag.2017.12.011

[3] Zamri MIP, Cordova F, Khairuddin ASM, Mokhtar N, Yusof R. Tree species classification based on image analysis using Improved-Basic Gray Level Aura Matrix. Comput Electron Agric. 2018; (124):227-233.

[4] doi:https://doi.org/10.1016/j.compag.2016.04.004

[5] Gazo R, Wells L, Krs V, Benes B. Validation of automated hardwood lumber grading system. Comput Electron Agric. 2018;(June):0-1. doi:10.1016/j.compag.2018.06.041

[6] Venkatachalapathy K, Sudhakar P. An Intelligent Recognition System for Identification of Wood Species. $J$ Comput Sci. 2014;10(7):1231-1237. doi:10.3844/jcssp.2014.1231.1237

[7] Guang-sheng C, Peng Z. Sensors and Actuators A : Physical Dynamic wood slice recognition using image blur information. Sensors Actuators A Phys. 2012;176:27-33. doi:10.1016/j.sna.2011.12.056

[8] Xiao B, Tang H, Jiang Y, Li W, Wang G. Brightness and contrast controllable image enhancement based on histogram specification. Neurocomputing. 2018;275:2798-2809. doi:10.1016/j. neucom. 2017.11.057

[9] Ratnasinga J, Ioras F, Swan TT, Yoon CY, Thanasegar G. Determinants of Occupational Accidents in the Woodworking Sector: The Case of the Malayasian Wooden Furniture

(C) The 2021 International Conference on Artificial Life and Robotics (ICAROB2021), January 21 to 24, 2021 
Industry. $\quad J \quad A p p l \quad S c i . \quad 2011 ; 11(3): 561-566$. doi:10.3923/jas.2011.561.566

[10] Wang Z. Applications of objective image quality assessment methods. Signal Process Mag IEEE. 2011;28(6):137-142. doi:10.1109/MSP.2011.942295

[11] Gulame M, Joshi KR, Kamthe RS. A Full Reference Based Objective Image Quality Assessment. Int $J$ Adv Electr Electron Eng. 2013;2(6):13-18

[12] Chandler DM. Seven Challenges in Image Quality Assessment: Past, Present, and Future Research. ISRN Signal Process. 2013;2013:1-53. doi:10.1155/2013/905685

[13] Khalid M, Lee ELY, Yusof R, Nadaraj M. Design of an intelligent wood species recognition system. Int J Simul Syst Sci Technol. 2008;9(3):9-19.

[14] Tou JY, Tay YH, Lau PY. A Comparative Study for Texture Classification Techniques on Wood Species Recognition Problem. In: 2009 Fifth International Conference on Natural Computation. IEEE; 2009:8-12. doi:10.1109/ICNC.2009.594

[15] Bremananth R.; Nithya B.; Saipriya R. Wood Species Recognition Using GLCM and Correlation. In: 2009 International Conference on Advances in Recent Technologies in Communication and Computing. Vol 51.; 2009:431-444. doi:https:/https:/10.1109/ARTCom.2009.10

[16] Wang Z, Simoncelli EP, Bovik AC. Multi-Scale Structural Similarity for Image Quality Assessment. In: Asilomar Conference on Signals, Systems and Computers. Vol 2. IEEE; 2003:1398-1402.

[17] Zhang L, Zhang D, Mou X. FSIM: a feature similarity index for image quality assessment. Image Process IEEE Trans. 2011;20(8):2378-2386

[18] Wang Z, Li Q. Information content weighting for perceptual image quality assessment. IEEE Trans Image Process. 2011;20(5):1185-1198. doi:10.1109/TIP.2010.2092435

[19] Xue W, Zhang L, Mou X, Bovik AC. Gradient magnitude similarity deviation: A highly efficient perceptual image quality index. Image Process IEEE Trans. 2014;23(2):684695.

[20] Meier E. The Wood Database. https://www.wooddatabase.com/. Published 2007. Accessed August 16, 2018.

[21] Rahman T, Haque MR, Rozario LJ, Uddin MS. Gaussian Noise Reduction in Digital Images Using a Modified Fuzzy Filter. In: International Conference on Computer and Information Technology (ICCIT). ; 2014:217-222.

[22] Rajagopal, H., Khairuddin, A.S.M., Mokhtar N et al. Application of image quality assessment module to motion-blurred wood images for wood species identification system. Wood Sci Technol. 2019:1-15.

[23] Mittal A, Moorthy AK, Bovik AC. No-Reference Image Quality Assessment in the Spatial Domain. IEEE Transactions on Image Processing. doi:10.1109/TIP.2012.2214050

[24] Heshalini Rajagopal, Norrima Mokhtar TFTMN, Izam WKWA. No-reference quality assessment for image- based assessment of economically important tropical woods. PLoS One. 2020:1-15. doi:10.1371/journal.pone.0233320

[25] Abd Latif, MH, Md. Yusof, H, Sidek, S.N, Rusli N. Implementation of GLCM Features in Thermal Imaging Affective State Detection. In: IEEE International Symposium on Robotics and Intelligent Sensors. Vol 76. ; 2015:308-315. doi:10.1016/j.procs.2015.12.298

[26] Rubiyah Yusof, Nenny Ruthfalydia Rosli MK. Using Gabor Filters as Image Multiplier for Tropical Wood Species Recognition System. In: International Conference on, Computer Modeling and Simulation, International Conference. ; 2010:289-294. doi:10.1109/UKSIM.2010.61

[27] Jain, Anil K. and FF. Unsupervised Texture Segmentation Using Gabor Filters. Pattern Recognit. 1991;24(12):11671186.

[28] Recommendation ITURBT (2002). 500-11. Methodology for the Subjective Assessment of the Quality of Television Pictures.; 2000.

[29] Chow LS, Rajagopal H, Paramesran R. Correlation between subjective and objective assessment of magnetic resonance (MR) images. Magn Reson Imaging. 2016;34(6):820-831. doi:10.1016/j.mri.2016.03.006

[30] Bindu K, Ganpati A, Sharma AK. A Comparative Study of Image Compression Algorithms. Int J Res Comput Sci. 2012;2(5):37-42. doi:10.7815/ijorcs.25.2012.046

[31] Li Sze Chow HR. Modified-BRISQUE as no reference image quality assessment for structural MR images. Magn Reson Imaging. 2017;43:74-87. doi:10.1016/j.mri.2017.07.016

[32] Chang, Chih-Chung and Lin C-J. LIBSVM: A library for support vector machines. ACM Trans Intell Syst Technol. 2011;2(3):27:1--27:27.

[33] Song X-K. Correlated Data Analysis: Modeling, Analytics, and Applications. Springer Science \& Business Media; 2007.

[34] Chai T, Draxler RR. Root mean square error (RMSE) or mean absolute error (MAE)? -Arguments against avoiding RMSE in the literature. Geosci Model Dev. 2014;7(3):1247-1250. doi:10.5194/gmd-7-1247-2014 